\section{Datenverarbeitungsbefugnisse der Anbieter von Diensten und Netzen}

Für Zugangsdienstanbieter und Netzanbieter enthalten $§ 307$ Abs. 2 S. 2 und Abs. 3 S. 3 SGB V neue Befugnisgrundlagen zur Datenverarbeitung ${ }^{247}$. Zugleich werden damit Zweckbeschränkungen geregelt. Daher dürfen Anbieter die personenbezogenen Daten nur für den Aufbau und Betrieb des Zugangsdienstes oder zum Zweck der Datenübertragung verarbeiten. Wegen des besonderen Schutzbedarfs der transportierten Inhaltsdaten wird jeweils die entsprechende Geltung des Fernmeldegeheimnisses gem. $\ 88$ TKG angeordnet ${ }^{248}$.

\section{IT- und Datensicherheit}

In der TI werden Gesundheitsdaten der Versicherten verarbeitetet. $\int 306$ Abs. 3 DSGVO betont, dass wegen des ,,besonderen Schutzbedarfs“ dieser Daten ein „hohes Schutzniveau“ gilt, was durch entsprechende technische und organisatorische Maßnahmen gem. Art. 32 DSGVO sicherzustellen ist. Wegen der technikneutralen Gesetzgebung werden im Einzelnen keine Maßnahmen vorgegeben ${ }^{249}$. Vielmehr werden in der Gesetzesbegründung allgemein die Schutzziele der Datensicherheit aufgelistet, z.B. Datenminimierung, Nichtverkettbarkeit oder Vertraulichkeit ${ }^{250}$. Weitere Aspekte der IT-Sicherheit werden in $\int \$ 329 \mathrm{ff}$. SGB V geregelt, wobei aufgrund der Kritikalität der TI eine Anlehnung an $\$ 8 \mathrm{a}$ Abs. 1 u. 3 BSIG erfolgt $t^{251}$. Flankierend wird in $\$ 395$ Abs. 2a SGB $V$ u. a. ein Verbot des In-Verkehr-Bringens und des Zur-Verfügung-Stellens von Komponenten oder Diensten der TI ohne Zulassung statuiert und der Bußgeldrahmen in §395 Abs. 3 SGB V deutlich erhöht. Damit soll der Abhän- gigkeit von der Sicherheit der TI und deren Diensten und Komponenten Rechnung getragen werden ${ }^{252}$.

\section{Zugriffsprotokollierung}

Die Protokollierung bei (versuchten) Zugriffen auf Anwendungen gem. \$334 Abs. 1 und 327 SGB V zum Zwecke der Datenschutzkontrolle wird nun in modifizierter Form in $\$ 309$ SGB V geregelt ${ }^{253}$. Die Protokolldaten, die erst nach drei Jahren zu löschen sind ${ }^{254}$, sollen dem Versicherten über die Benutzeroberfläche eines geeigneten Endgeräts gem. $\$ 342$ Abs. 2 Nr. 1 lit. d, Nr. 2 lit. g SGB V zur Verfügung gestellt werden $^{255}$. Weitere Regelungen zur Zugriffsprotokollierung enthält für eVerordnungen $\$ 361$ Abs. 2 S. 2 SGB V und für Zugriffe auf andere Anwendungen $\$ 339$ Abs. 3 S. 2, Abs. 5 S. 2 SGB V. Auch hier gewährleistet die Protokollierungspflicht, dass der Versicherte seine Rechte im Rahmen der Patientensouveränität wahrnehmen und kontrollieren $\mathrm{kann}^{256}$.

Der Beitrag wird in Heft 1/2021 fortgesetzt.

247) BT-Dr. 19/18793, S. 101

248) \307 Abs. 2 S. 3 und Abs. 3 S. 4 SGB V, BT-Dr. 19/18793, S. 101 249) BT-Dr. 19/18793, S. 99.

250) S. dazu Sohr/Kemmerich, in: Kipker, Cybersecurity, Rechtshandbuch, 2020, Kap. 2, Rdnrn. 6ff.; Dochow, Telematik im Gesundheitswesen, 2017, S. 789 ff. m. w. N

251) Vgl. BT-Dr. 19/18793, S. 107.

252) Vgl. BT-Dr. 19/18793, S. 136.

253) Bisher $₫ 291$ a Abs. 6 S. 3-5 SGB V a.F.

254) $\$ 309$ Abs. 1, Abs. 3 SGB V i. V. mit $\$ 195$ BGB.

255) S. a. $₫ 312$ Abs. 6 SGB V.

256) S. BT-Dr. 19/18793, S. 102, 110, 130.

\title{
Intelligente Medizinprodukte: Ist der geltende Rechtsrahmen noch aktuell?
}

\section{Katrin Helle}

Künstliche Intelligenz (nachfolgend auch KI) eröffnet der Medizin völlig neue Möglichkeiten. Die ersten Medizinprodukte, die sich KI bedienen, sind auf dem Markt. Neben die Chancen des Einsatzes von KI in gesamtgesellschaftlicher Hinsicht treten Rechtsfragen der Regulierung einer selbstlernenden, sich ständig entwickelnden und autonom entscheidenden Software sowie der Schadenshaftung, falls sich die Software einmal irren sollte, und angesichts des notwendigen Einsatzes von „Big Data“ nicht zuletzt des Datenschutzes. Diesen Rechtsfragen widmet sich der nachfolgende Beitrag.

\section{Einführung}

Immer mehr Medizinprodukte nutzen KI, um seltene Krankheiten zu erkennen, Diagnosen in Sekundenschnelle zu stellen und Patienten präziser behandeln zu können. Beim Erkennen von Krankheiten ist ihre Trefferquote unter

Rechtsanwältin Dr. iur. Katrin Helle (née Rübsamen),

Dierks+Company Rechtsanwaltsgesellschaft mbH, HELIX HUB, Invalidenstraße 113, 10115 Berlin, Deutschland bestimmten Bedingungen sogar besser als die von Ärzten. Die wichtige Frage, ob die heute geltenden, auf statische Produkte und deterministische Software ausgerichteten Rechtsvorschriften den Besonderheiten und Risiken der KI gewachsen oder neue Regelungen erforderlich sind, beschäftigte jüngst die Europäische Kommission in ihrem Weißbuch zur KI und dem Bericht über die Auswirkungen von KI in Hinblick auf Sicherheit und Haftung ${ }^{1}$. Es folgte

1) Europäische Kommission, Weißbuch, Zur Künstlichen Intelligenz - ein europäisches Konzept für Exzellenz und Vertrauen, $\operatorname{COM}(2020) 65$ (nachfolgend Weißbuch); Bericht über die Auswirkungen Künstlicher Intelligenz, des Internets der Dinge und der Robotik in Hinblick auf Sicherheit und Haftung, COM(2020)64 (nachfolgend Kommissionsbericht); beide v. 19.2.2020. Weißbuch und Kommissionsbericht setzen den Einsatz einer hochrangigen Expertengruppe für KI (HEG-KI) fort, die eine Definition der KI entwickelte (s. Definition der Künstlichen Intelligenz: Wichtigste Fähigkeiten und Wissenschaftsgebiete v. 8.4.2019, nachfolgend HEG-KI Definition) und einen Bericht zur Haftung für KI verfasste (s. Liability for Artificial Intelligence and other emerging digital technologies v. 21.11.2019, nachfolgend HEG-KI Bericht zur Haftung). 
kürzlich ein Berichtsentwurf des Europäischen Parlaments über die zivilrechtliche Haftung beim Einsatz von $\mathrm{KI}^{2}$.

Obwohl Medizinprodukte, die auf KI basieren, bereits für den Vertrieb durch die U.S. Food and Drug Administration (FDA) zugelassen wurden ${ }^{3}$, sind diverse rechtliche Fragen ungeklärt. Je größer das Entwicklungspotenzial intelligenter Medizinprodukte, Menschen in die Lage zu versetzen, noch nicht erreichte Intelligenz zu entwickeln und zur Lösung einiger der größten Herausforderungen der Menschheit beizutragen, beispielsweise der Vorhersage von Krankheitsausbrüchen und Virusverbreitung ${ }^{4}$, umso bedeutender wird die Evaluierung ihrer rechtlichen Rahmenbedingungen. Mit ihren Veröffentlichungen haben Kommission und Parlament einen wichtigen Diskurs der Interessenträger angestoßen, dessen Stand der nachfolgende Beitrag im Überblick aufzeigt.

Hierbei wird unter KI eine Software verstanden, die in Bezug auf ein vorgegebenes Ziel Daten erfasst und interpretiert, Schlussfolgerungen daraus zieht oder abgeleitete Informationen verarbeitet und über das bestmögliche Handeln zur Erreichung des vorgegebenen Ziels entscheidet, wobei die Software in der Lage ist, die Auswirkungen früherer Handlungen auf die Umgebung zu analysieren und ihr Verhalten entsprechend anzupassen. Hierfür nutzt die Software große Mengen an Daten, die sie selbstständig auswertet $^{5}$. KI ist zu abstrahierendem Denken im Wege des Vergleichens fähig und durch sog. Deep Learning in der Lage, sich unabhängig von ihrer menschlichen Programmierung zu entwickeln ${ }^{6}$. In der Medizin kann durch den Einsatz von KI zur Unterstützung ärztlicher Entscheidungen aus Hardware, Komponenten oder Software ein ,,intelligentes" Medizinprodukt werden.

\section{Rechtliche Rahmenbedingungen}

Die rechtlichen Rahmenbedingungen für intelligente Medizinprodukte finden sich insbesondere im Medizinprodukterecht, Haftungsrecht und Datenschutzrecht.

\section{Medizinprodukterecht}

$\mathrm{Ob}$ KI der medizinprodukterechtlichen Regulierung unterliegt, richtet sich gegenwärtig nach dem Medizinproduktegesetz (MPG) $)^{7}$. Mit Wirkung v. 26.5.2021 gelten anstelle dessen die Medizinprodukteverordnung (MDR) ${ }^{8}$ und das Medizinprodukterecht-Durchführungsgesetz (MPDG). Ab dem 26.5.2022 wird ferner die In-vitro-Diagnostika Verordnung (IVDR) ${ }^{9}$ gelten. Im Weiteren geht der Beitrag schwerpunktmäßig auf die künftige Rechtslage ein. Auslegungshilfen enthalten die rechtlich unverbindlichen Leitlinien der Koordinierungsgruppe Medizinprodukte zur Qualifizierung und Klassifizierung von Software gemäß MDR und IVDR ${ }^{10}$ sowie das von der Kommission veröffentlichte Handbuch einer Expertengruppe zur Abgrenzung und Klassifizierung von Medizinprodukten ${ }^{11}$.

\section{a) Software als Medizinprodukt}

KI-basierte Software ist als Medizinprodukt einzuordnen, wenn sie eigenständig einen der in Art. 2 Nr. 1 MDR genannten spezifischen medizinischen Zweck erfüllt, wobei es nicht darauf ankommt, ob die Software, etwa mittels Sensoren, ,im oder am menschlichen Körper“ wirkt ${ }^{12}$. Die Zweckbestimmung trifft der Hersteller in der Kennzeichnung, Gebrauchsanweisung oder den Werbe- und Verkaufsmaterialien (Art. 2 Nr. $12 \mathrm{MDR} / \mathrm{IVDR}$ ). Software, die medizinische Informationen erzeugt, verarbeitet, verändert oder analysiert, ist danach als Medizinprodukt einzustufen, sofern dies zur Erfüllung eines medizinischen Zwecks $\operatorname{erfolgt}^{13}$. Das gilt etwa für eine Software, die auf Grundlage bestimmter Kriterien (z.B. Alter) und eines fötalen Ultraschallbilds das Risiko einer Trisomie 21 bestimmt ${ }^{14}$.
Dagegen fallen Informationssysteme, die sich auf die bloße Speicherung, Wiedergabe oder den Transfer von Daten beschränken, nicht unter die Medizinprodukteregulierung ${ }^{15}$.

Von der als Medizinprodukt einzuordnenden Software ist solche Software abzugrenzen, die (Hardware) Medizinprodukte steuert oder deren Anwendung beeinflusst, aber weder einen eigenständigen spezifischen medizinischen Zweck verfolgt noch eigenständig Informationen für einen medizinischen Zweck generiert. Solche Software ist ein Bestandteil des (Hardware) Medizinprodukts oder kann als Zubehör gelten (Art. 2 Nr. 2 MDR/Art. 2 Nr. 4 IVDR) ${ }^{16}$.

Sofern also KI zur eigenständigen Erfüllung eines spezifischen medizinischen Zwecks eingesetzt wird, gilt sie nach der hiesigen Einordnung als intelligentes Medizinprodukt. Verfolgt ein KI-gestütztes (Hardware) Medizinprodukt den spezifischen medizinischen Zweck, ist jenes das unter die MDR oder IVDR fallende intelligente Medizinprodukt; die KI-Software ist mittelbar über das (Hardware) Medizinprodukt reguliert ${ }^{17}$.

\section{b) Zertifizierung von intelligenten Medizinprodukten}

In der EU existieren bislang keine speziellen regulatorischen Rahmenbedingungen und Verfahren, die die Besonderheiten von KI in Medizinprodukten adressieren. Es gilt daher der Rechtsrahmen für Software als Medizinprodukt. Die MDR und IVDR beinhalten für Software zwar vereinzelt besondere Anforderungen, sind jedoch nicht darauf ausgelegt, dass die einem intelligenten Medizinprodukt zugrundeliegenden Algorithmen kontinuierlich lernen und sich selbstständig weiterentwickeln sowie autonom entscheiden.

2) Europäisches Parlament, Entwurf eines Berichts mit Empfehlungen an die Kommission zu zivilrechtlicher Haftung beim Einsatz künstlicher Intelligenz v. 27. 4. 2020, 2020/2014(INL).

3) U.a. ein Gerät, das eine Retinopathie bei Diabetespatienten erkennt, https://www.fda.gov/news-events/press-announcements/ fda-permits-marketing-artificial-intelligence-based-device-detect-certain-diabetes-related-eye, sowie eine Software, die Diagnosen von Handgelenksfrakturen unterstützt, https://www.fda. gov/news-events/press-announcements/fda-permits-marketingartificial-intelligence-algorithm-aiding-providers-detectingwrist-fractures, Zugriff am 23.8.2020.

4) S. Kommissionsbericht, S. 3. Vgl. zur Vorhersage der Verbreitung des SARS-CoV-2 Virus durch KI https://www.wired.com/ story/ai-epidemiologist-wuhan-public-health-warnings/, Zugriff am 23.8.2020.

5) Vgl. HEG-KI Definition, S. 6

6) Zur Funktionsweise lernender Algorithmen in der Medizin näher Katzenmeier, MedR 2019, 259, 268.

7) Das MPG setzt die Medizinprodukterichtlinie 93/42/EWG, geänd. durch Richtlinie 2007/47/EG, um.

8) Verordnung (EU) 2017/745 über Medizinprodukte, geänd. durch Berichtigungen ABl. EU L 117 v. 3.5.2019, S. 9 und ABl. EU L 334 v. 27.12.2019, S. 165 sowie Verordnung (EU) 2020/561 v. 23. 4. 2020 .

9) Verordnung (EU) 2017/746 über In-vitro-Diagnostika, geänd. durch Berichtigungen ABl. EU L 117 v. 3. 5.2019, S. 11 und ABl. EU L 334 v. 27.12.2019, S. 167.

10) MDCG 2019-11 Guidance on Qualification and Classification of Software in Regulation (EU) 2017/745 - MDR and Regulation (EU) 2017/746 - IVDR aus Oktober 2019 (nachfolgend MDCG Guidance).

11) EU Manual on Borderline Classification in the Community Regulatory Framework for Medical Devices, Version 1.22 (052019), zu Software, S. $77 \mathrm{ff}$.

12) Vgl. EuGH, EuZW 2018, 166, 167 f., Rdnrn. 23 f., 28 ff., 39.

13) MDCG Guidance, S. 6.

14) MDCG Guidance, S. 7.

15) Vgl. EuGH, EuZW 2018, 166, 167, Rdnrn. 26, 33; MDCG Guidance, S. 8.

16) S. MDCG Guidance, S. 5, 8 (,,Software driving or influencing the use of a device").

17) S. MDCG Guidance, S. 8. 
aa) Wesentliche Anforderungen an Medizinprodukte Nachfolgend werden wesentliche Anforderungen benannt, die bei der Entwicklung und Zertifizierung sowie der Produktbeobachtung intelligenter Medizinprodukte angesichts ihrer besonderen Merkmale, insbesondere der Opazität, Komplexität, Unvorhersehbarkeit und Autonomie ${ }^{18}$ erhöhte Aufmerksamkeit verlangen.

Medizinprodukte dürfen in Verkehr gebracht und in Betrieb genommen werden, wenn sie eine CE-Kennzeichnung tragen. Die CE-Kennzeichnung ist an die Erfüllung der in Anhang I MDR/IVDR festgelegten grundlegenden Sicherheits- und Leistungsanforderungen und das Durchlaufen eines Konformitätsbewertungsverfahrens sowie die Ausstellung einer Konformitätserklärung seitens des Herstellers geknüpft (Art. 5, Art. 10 Abs. 5 bzw. 6 MDR/ IVDR). Die Klassifizierung des Produkts bestimmt das einschlägige Konformitätsbewertungsverfahren ${ }^{19}$. Die grundlegenden Sicherheits- und Leistungsanforderungen eines Medizinprodukts mit einem Softwarebestandteil wie auch eines (reinen) Software-Medizinprodukts umfassen u.a., dass die Wiederholbarkeit, Zuverlässigkeit und Leistung gewährleistet sind ${ }^{20}$. Bei der Entwicklung und Herstellung sind ferner die Grundsätze des SoftwareLebenszyklus, des Risikomanagements einschließlich der Informationssicherheit, der Verifizierung und der Validierung zu berücksichtigen ${ }^{21}$.

Die vorgenannten Kriterien dürften sich bei intelligenten Medizinprodukten als Herausforderung darstellen. So soll sich eine KI gerade weiterentwickeln und ist nicht auf die Wiederholung ihrer Leistungen ausgelegt. Selbstlernende Systeme passen sich während ihres Einsatzes ständig an neue Erkenntnisse an. Dies kann die Funktionsweise im weiteren Verlauf des Lebenszyklus ändern und zu Risiken führen, die zum Zeitpunkt des Inverkehrbringens nicht bestanden ${ }^{22}$. Ein in Betrieb befindliches intelligentes Medizinprodukt könnte sich erheblich von dem Produkt unterscheiden, das das Konformitätsbewertungsverfahren durchlaufen hat. Ferner dürften die Verifizierung und Validierung bei einem zumindest teilautonom entscheidenden System, dessen Entscheidungsprozesse schwer überprüfbar sind, schwierig bis unmöglich sein (Blackbox-Problema$\mathrm{tik}^{23}$. Das wirft die Frage auf, in welchem Umfang Änderungen infolge der ständigen Anpassung des intelligenten Produkts noch durch die bestehende Konformitätsbewertung abgedeckt sind ${ }^{24}$ bzw. ab wann es einer neuen Konformitätsbewertung bedarf, um eine konsistente Einhaltung der Funktionalität und Sicherheit des intelligenten Produkts zu gewährleisten. Mit der Beantwortung dieser Frage wird sich der europäische Gesetzgeber zu befassen haben.

Des Weiteren muss der Hersteller bei Inverkehrbringen eines Medizinprodukts eine Produktkennung zuteilen ${ }^{25}$. Ändern sich die ursprüngliche Leistung, die Sicherheit oder die bestimmungsgemäße Verwendung einer Software oder die Auswertung der Daten durch eine Software nicht nur geringfügig, wird eine erneute UDI-DI erforderlich, wobei zu den Änderungen auch neue oder geänderte Algorithmen gehören ${ }^{26}$. Weil intelligente Medizinprodukte gerade darauf ausgelegt sind, sich stetig weiterzuentwickeln, hat das naturgemäß Änderungen an ihrer Leistung und der Auswertung der Daten zufolge. Sofern es für KI bei diesen regulatorischen Anforderungen verbleibt, sollten Hersteller intelligenter Medizinprodukte darauf achten, dass sie erforderlichenfalls rechtzeitig eine neue UDI-DI zuteilen.

\section{bb) Regulierungsansätze im Umgang}

mit intelligenten Medizinprodukten

Kommission und Bundesregierung sind einhellig der Meinung, dass für KI-gestützte Produkte auf bestehende Konformitätsbewertungsmechanismen zurückgegriffen werden kann. Lediglich die materiellen Anforderungen, hier also des Medizinprodukterechts, müssten angepasst werden ${ }^{27}$.
Angesichts des erheblichen Interesses der Allgemeinheit an einem zügigen Einsatz intelligenter Medizinprodukte wird eine „Fast-Track-Zulassung“ für erwägenswert gehalten ${ }^{28}$.

Den vorgenannten regulatorischen (materiellen) $\mathrm{He}$ rausforderungen hinsichtlich intelligenter Medizinprodukte könnte schon auf Grundlage des geltenden Rechts begegnet werden, indem man auf bestimmte Maßnahmen (Schlüsselmerkmale) besonderen Wert legt, die das Weißbuch aufzeigt ${ }^{29}$. Hierzu zählen Anforderungen an

- die Trainings-, Test- und Evaluierungsdaten, deren Auswahl in Bezug auf inhaltliche Korrektheit, Aktualität, Repräsentativität und Vollständigkeit gewährleisten soll, dass die Nutzung der KI-gestützten Produkte sicher ist,

- die Aufbewahrung von Daten und Aufzeichnungen über die Programmierung des Algorithmus, um Entscheidungen des Systems i.S. der Transparenz, Nachvollziehbarkeit und Erklärbarkeit zurückverfolgen und überprüfen zu können,

- die Bereitstellung angemessener Informationen, u.a. von Angaben über Fähigkeiten und Grenzen der KI,

- die Robustheit und Genauigkeit des Systems in allen Phasen des Lebenszyklus, die Reproduzierbarkeit seiner Ergebnisse, die Fähigkeit, Fehler und Unstimmigkeiten bewältigen zu können und eine Widerstandsfähigkeit gegen Angriffe und Manipulationen ${ }^{30}$ sowie

- die Überwachung des KI-Systems während seines Betriebs $^{31}$ (Produktbeobachtungspflicht) und die Möglichkeit menschlichen Eingreifens.

Die Kommission hält darüber hinaus bei sich weiterentwickelnden und lernfähigen KI-Systemen mit hohem Risiko das Erfordernis wiederholter Bewertungen während der Lebensdauer des Systems für möglich ${ }^{32}$. Die Bundesregierung verortet diese Frage unter dem Gesichtspunkt der stärkeren Verknüpfung der Rechtsbereiche Bereitstellung von Produkten und Betrieb ${ }^{33}$. $\mathrm{Ob}$ und unter welchen Anforderungen das Durchlaufen eines vollständigen Konformitätsbewertungsverfahrens für intelligente Medizinprodukte

18) S. Weißbuch, S. 14; Kommissionsbericht, S. 2.

19) Vgl. Art. 51, 52 MDR/Art. 47, 48 IVDR. Die Klassifizierung von Software erfolgt nach Anhang VIII, Regel 11 MDR. Abhängig davon, ob Software dazu bestimmt ist, Informationen zu liefern, die zu Entscheidungen für diagnostische oder therapeutische Zwecke herangezogen werden, und welche Auswirkungen die Entscheidungen haben können, gehört sie zur Klasse IIa, IIb oder III.

20) Anhang I, Abschn. 17.1 MDR.

21) Anhang I, Abschn. 17.2 MDR. Dazu Dettling, PharmR 2019, 633, 637.

22) Das gilt insbesondere für Produkte, die häufige SoftwareUpdates benötigen oder auf maschinellem Lernen beruhen. S. Weißbuch, S. 16

23) Vgl. Dettling, PharmR 2019, 633, 637; Katzenmeier, MedR 2019, 259, 269.

24) Dazu Anhang VII, Abschn. 4.9 MDR.

25) Vgl. Art. 27 MDR/Art. 24 IVDR: Unique Device Identification-Device Identifier (UDI-DI).

26) Anhang VI, Teil C Abschn. 6.5.2., 6.5.3 MDR.

27) Weißbuch, S. 27f.; BReg., Stellungnahme zum Weißbuch v. 29.6.2020 (nachfolgend BReg.-SN Weißbuch), S. 18.

28) S. Hacker, NJW 2020, 2142, 2145.

29) Weißbuch, S. 22ff. Siehe auch Kommissionsbericht, S. 8 ff.; BReg.-SN Weißbuch, S. $11 \mathrm{ff}$.

30) Beispiele nennt der Kommissionsbericht, S. 6.

31) Laut BReg. darf der Lernprozess der KI nicht unkontrolliert erfolgen, die menschliche Aufsicht müsse die Nachvollziehbarkeit gewährleisten, BReg.-SN Weißbuch, S. 24

32) Weißbuch, S. 28. So auch die BReg., BReg.-SN Weißbuch, S. 17f. Laut Kommission kommt das in Betracht, wenn es zu einer erheblichen Änderung, etwa der Produktfunktion kommt, die in der ursprünglichen Risikobewertung nicht vorhersehbar war, Kommissionsbericht, S. 8 f.

33) BReg.-SN Weißbuch, S. 22. 
zu erfolgen hat, bleibt der Entscheidung des europäischen Gesetzgebers vorbehalten. Der Diskurs hierzu wird ggf. anhand der FDA Regulierungsansätze (dazu sogleich) geführt. Die behördliche Uberwachung intelligenter Medizinprodukte während des Betriebs könnte durch erhöhte Dokumentations- und Offenlegungspflichten der Hersteller und Anwender ermöglicht und erleichtert werden ${ }^{34}$.

c) Exkurs: US-amerikanische Regulierungsansätze

Die FDA veröffentlichte Anfang 2019 einen Vorschlag für eine an die Funktionsweise von KI in Medizinprodukten angepasste Bewertungs- und Zulassungspraxis ${ }^{35}$. Um die Zulassung adaptiver und kontinuierlich lernender Verfahren der KI zu erleichtern und so ihre Entwicklung zu fördern, schlägt die FDA in ihrem Entwurf einen den gesamten Lebenszyklus des Produkts begleitenden regulatorischen Ansatz vor.

Der FDA Ansatz fordert ein Qualitätsmanagementsystem des Herstellers, das auf die Gewährleistung einer hohen Qualität während des gesamten Lebenszyklus des Produkts abzielt. Ferner soll der Hersteller mit einem ,,predetermined change control plan“ (Plan) die auf Grundlage der lernenden Softwarealgorithmen antizipierten Modifikationen des Produkts sowie die Methoden zur Erreichung der Modifikationen und zur Begrenzung der damit verbundenen Risiken offenlegen können. Mit Modifikationen des Produkts während seines Lebenszyklus wird sodann wie folgt umgegangen: Auf Grundlage und in den Grenzen eines genehmigten Plans kann die FDA von dem Erfordernis einer Neuzulassung absehen und lediglich eine Dokumentationspflicht für Änderungen regeln. Liegen die Modifikationen außerhalb des genehmigten Plans, führt die FDA einen „,focused review“ durch, sofern der Verwendungszweck des Produkts nicht verändert wird, anderenfalls muss ein neues Genehmigungsverfahren durchlaufen werden. Der FDA Ansatz fordert ferner Transparenz und die Ermöglichung der Überwachung der Leistungsfähigkeit der Produkte durch regelmäßige Berichte an die FDA. Des Weiteren sind Anwender über etwaige Änderungen und deren Auswirkungen $z u$ informieren.

Der FDA Ansatz hat den Vorteil, dass der Hersteller nicht bei jeder Änderung an einem intelligenten Medizinprodukt ein erneutes komplettes Bewertungsverfahren zu durchlaufen hätte. Soweit ersichtlich, sind die Rückmeldungen zum Vorschlag bis heute nicht in eine verbindliche Leitlinie oder Entscheidung der FDA eingeflossen.

\section{Haftung}

Intelligente Medizinprodukte bergen besondere Fehlerquellen, die auf fehlerhaften Algorithmen, elektronischen Viren oder der KI Besonderheit des Erlernens neuer Funktionen nach einem System von Versuch und Irrtum bzw. Fehler beruhen können. Weder der europäische noch der nationale Gesetzgeber haben für Medizinprodukte ein spezielles Haftungssystem geschaffen, so dass die allgemeinen Regelungen gelten. Neben der Haftung des Herstellers eines intelligenten Medizinprodukts kommt eine Verantwortlichkeit des Anwenders in Betracht, oftmals des behandelnden Arztes.

\section{a) Haftung des Herstellers}

Die Haftung des Herstellers mangelhafter intelligenter Medizinprodukte kann auf mehreren Grundlagen beruhen. Ist der Hersteller Vertragspartner des Geschädigten, haftet er nach Gewährleistungsrecht. Liegen dem Vertrag Allgemeine Geschäftsbedingungen zugrunde, ist die Wirksamkeit von Haftungsausschlüssen durch $\$ 309$ Nr. 7 BGB begrenzt. Für Fehler bzw. die Verletzung von Verkehrssicherungspflichten ist der Hersteller darüber hinaus nach den allgemeinen deliktischen Grundsätzen der Produkt- bzw.
Produzentenhaftung verantwortlich, also nach dem ProdHaftG und den $\int S 823 \mathrm{ff}$. BGB.

aa) Haftung nach Produkthaftungsgesetz

In Umsetzung der Produkthaftungsrichtlinie 85/374/EWG normiert $\$ 1$ Abs. 1 ProdHaftG eine verschuldensunabhängige Haftung des Herstellers eines fehlerhaften Produkts für die Verletzung einer Person oder die Beschädigung einer dem privaten Gebrauch dienenden Sache, wenn sie auf der Fehlerhaftigkeit des Produkts beruht. Noch ungeklärt ist, ob trägerlos übermittelte, nicht in eine Sache integrierte Software die Eigenschaft der ,,beweglichen Sache“ besitzt (vgl. $\$ 2$ ProdHaftG) ${ }^{36}$. Die Kommission und Bundesregierung haben eine normative Klarstellung angekündigt, dass auch nicht integrierte Software in den Anwendungsbereich der Produkthaftung fallen soll ${ }^{37}$. Bis dahin ist $\$ 2$ ProdHaftG nach teilweise vertretener Auffassung analog auf Software anzuwenden ${ }^{38}$

Ein Produkt ist gemäß $₫ 3$ ProdHaftG fehlerhaft, wenn es nicht die Sicherheit gebietet, die unter Berücksichtigung aller Umstände berechtigterweise erwartet werden kann. Die Pflichten des Herstellers bestimmen sich nach dem aktuellen Stand der Wissenschaft zum Zeitpunkt des Inverkehrbringens des Produkts ( $\$ 3$ Abs. 1 Buchst. c, Abs. 2 ProdHaftG). Aufgrund des Risikopotenzials im Bereich der Medizinprodukte gelten hohe Sicherheitsanforderungen $^{39}$. Schon bei der Konstruktion müssen die erhöhten Anforderungen speziell an Software beachtet werden: Gefordert sind eine sorgfältige Programmierung am Maßstab des aktuellen Stands von Wissenschaft und Technik ${ }^{40}$ sowie für den Fall des Auftretens von Defekten Vorkehrungen zum Zwecke der Risikobegrenzung und des Erhalts der Leistungsfähigkeit ${ }^{41}$. Bei der Festlegung der Sicherheitsstandards sind neben der bestimmungsgemäßen Verwendung die vorhersehbare Verwendung, wie auch vorhersehbare Fehlbedienungen und Zweckentfremdungen durch den Anwender einzukalkulieren und ausreichende Hinweise und Warnungen zur Abwendung der Haftung erforderlich $^{42}$. Ist das intelligente Medizinprodukt fähig, neue Funktionen zu erlernen, muss in der mit dem Medizinprodukt bereitgestellten Gebrauchsanweisung eine entsprechende Instruktion an den Anwender erfolgen, sofern nicht die neue Funktion selbsterklärend ist ${ }^{43}$.

Hinsichtlich KI sind zwei Ausschlussgründe des $₫ 1$ Abs. 2 ProdHaftG relevant: Danach besteht keine Haftung, wenn davon auszugehen ist, dass der für den Schaden ursächliche

34) Vgl. Art. 93 ff. MDR/Art. 88 ff. IVDR. S. Weißbuch, S. 28; BReg.-SN Weißbuch, S. 23; Hacker, NJW 2020, 2142, 2143 f.

35) Proposed Regulatory Framework for Modifications to Artificial Intelligence/Machine Learning (AI/ML)-Based Software as a Medical Device (SaMD). Abrufbar unter https://www.fda. gov/files/medical\%20devices/published/US-FDA-ArtificialIntelligence-and-Machine-Learning-Discussion-Paper.pdf; die Rückmeldungen sind abrufbar unter https://www.regulations. gov/docketBrowser? $\mathrm{rpp}=25 \& \mathrm{so}=\mathrm{DESC} \& \mathrm{sb}=$ commentDueDate $\& p o=0 \& D=F D A-2019-N-1185 ;$ Zugriff am 23. 8. 2020.

36) U.a. Ortner/Daubenbüchel, NJW 2016, 2918, 2919 und Jakobs/ Huber, MPR 2019, 1, $2 \mathrm{f}$. verneinen die Produkteigenschaft. Zum Meinungsspektrum Seehafer/Kohler, EuZW 2020, 213, 214; Oechsler, in: Staudinger, 2018, \$2 ProdHaftG, Rdnrn. $64 \mathrm{ff}$; Reusch, in: Kaulartz/Braegelmann (Hrsg.), Rechtshandbuch Artificial Intelligence und Machine Learning, 2020, S. 114 f. Vgl. auch Katzenmeier, MedR 2019, 259, 265.

37) Kommissionsbericht, S. 17. S. BReg.-SN Weißbuch, S. 24f.; HEG-KI, Bericht zur Haftung, S. 6, Rdnr. 13.

38) Vgl. Hacker, NJW 2020, 2142, 2145 m.w. N.

39) Vgl. u.a. Anhang I, MDR/IVDR, SS $11 \mathrm{f}$. MPDG.

40) BGH, NJW 2009, 2952, 2953

41) S. Anhang I, Abschn. 17.1 MDR.

42) Vgl. Kommissionsbericht, S. 7 und Fn. 35; Oechsler, in: Staudinger 2018, \$3 ProdHaftG, Rdnrn. 56 ff.

43) S. Anhang I, Abschn. 23.1, 23.4 MDR. 
Fehler bei Inverkehrbringen des Produkts noch nicht bestand (Nr. 2), oder der Fehler nach dem Stand der Wissenschaft und Technik zum Zeitpunkt des Inverkehrbringens nicht erkannt werden konnte (Nr. 5). Sollte das intelligente Medizinprodukt autonom in dem Sinne handeln, dass es sich eine fehlerhafte Funktion selbst beibringt, dürfte der Ausschluss des später aufgetretenen Fehlers oftmals eingewendet werden; entsprechendes gilt für den Ausschluss des Entwicklungsrisikos, soweit Erkenntnisse zur Funktionsweise von KI fehlen und ihre Entscheidungen nicht vorhergesehen werden können ${ }^{44}$. Die Beweislast für Fehler, Schaden und den kausalen Zusammenhang zwischen den beiden Elementen trägt der Geschädigte, während der Hersteller etwaige Ausschlussgrüne zu beweisen hat ( $\$ 1$ Abs. 4 ProdHaftG). Aufgrund der Komplexität der Materie und der beschränkten Nachvollziehbarkeit der Entscheidungsprozesse der KI ist ein Beweis für den Geschädigten in vielen Fällen erschwert, nur mit Hilfe eines Sachverständigen zu erbringen oder gar unmöglich ${ }^{45}$.

Die Besonderheiten der KI haben zu Diskussionen über eine Anpassung des Haftungssystems bis hin zur Einführung eines neuen Haftungsregimes zumindest für KI-Systeme mit Schädigungspotenzial geführt ${ }^{46}$.

Hält der Hersteller nicht die regulatorischen Vorgaben an das intelligente Produkt ein, begründet dies nach geltendem Recht bereits den Anschein der Fehlerhaftigkeit des Produkts $^{47}$ und könnte künftig sogar eine Beweislastumkehr bewirken ${ }^{48}$. Für eine Haftung des Herstellers selbst für gefahrenträchtiges Verhalten der KI aufgrund einer nach dem Inverkehrbringen unvorhergesehen erlernten Funktion wird zum Teil auf sein Wissen um die Risiken des autonom lernenden Produkts verwiesen, das der Hersteller in die Bewertung der Fehlerhaftigkeit einzubeziehen habe ${ }^{49}$. Teilweise wird gefordert, dass die Ausschlussgründe des später aufgetretenen Fehlers und des Entwicklungsrisikos nicht oder nur sehr eingeschränkt Anwendung finden sollen, weil der Entwickler um die Lernfähigkeit seiner Software weiß und sie bewusst hervorruft ${ }^{50}$ bzw. es sich um eine programmierte Nichtprognostizierbarkeit der Algorithmus-Entscheidungen handelt ${ }^{51}$. Die Kommission erwägt, von der Bundesregierung unterstützt, eine Änderung des Verständnisses des ,Zeitpunkts des Inverkehrbringens“52. Der Hersteller könnte danach für das Aufspielen eines fehlerhaften Updates oder das Unterlassen einer Korrektur fehlerhafter Funktionen haftbar sein, die die KI autonom erlernt hat. Das käme der Einführung einer Produktbeobachtungspflicht gleich, die dem Produkthaftungsrecht heute fremd ist. Gefordert wird teilweise die Schaffung einer Gefährdungshaftung für den Hersteller KI-gestützter Produkte ${ }^{53}$, wobei seitens der Bundesregierung Zurückhaltung gegenüber einer generellen produktunabhängigen Gefährdungshaftung besteht ${ }^{54}$. Weil das Risiko digitaler Entscheidungsautonomie anders gelagert sei als die durch die herkömmlichen Gefährdungshaftungen abgedeckten Risiken, etwa im Straßenverkehr, soll es sich nach einer Ansicht bei KI-basierten Produkten um eine ,digitale Assistenzhaftung“ nur für rechtswidrige autonome Fehlentscheidungen der Software handeln ${ }^{55}$. Darüber hinaus wird eine gesetzliche Versicherungspflicht gegen Schäden durch KI diskutiert ${ }^{56}$.

Hinsichtlich der Beweislast zieht die Kommission eine Beweislastumkehr zugunsten der Patienten in Erwägung ${ }^{57}$, sofern eine Verpflichtung des Herstellers nicht ausreichen sollte, die relevanten Daten aufzuzeichnen und zur Verfügung zu stellen (Befundsicherungspflicht ${ }^{58}$. Die Bundesregierung äußert sich zurückhaltend zu einer Änderung der Beweislastverteilung ${ }^{59}$. Unter dem Gesichtspunkt des angemessenen Ausgleichs der berechtigten Interessen von potentiell Geschädigten und Herstellern wird schließlich gefordert $\mathrm{zu}$ berücksichtigen, ob der Hersteller funktionserhaltende Sicherheitsaktualisierungen für das intelligente Medizinprodukt bereitstellt und den Geschädigten hiervon in Kenntnis setzt. Unterlässt der Geschädigten das Aufspielen des Updates, käme eine Haftungsminderung in Betracht ${ }^{60}$.

\section{bb) Haftung nach $\$ \int 823 \mathrm{ff}$. BGB}

Neben der Produkthaftung kann das Inverkehrbringen eines mit einem Konstruktions- oder Fabrikationsfehler behafteten intelligenten Medizinprodukts oder die Missachtung der Instruktions- oder Produktbeobachtungspflicht eine verschuldensabhängige Haftung des Herstellers nach \823 Abs. 1 BGB begründen ${ }^{61}$. Den Hersteller medizinischer Software treffen wie im Bereich der Produkthaftung spezifische Verkehrssicherungspflichten ${ }^{62}$. Es gelten auch hier die erhöhten Sicherheitsanforderungen aus dem $\mathrm{Me}-$ dizinprodukterecht. Hinsichtlich der Konstruktion intelligenter Medizinprodukte sowie der erforderlichen Hinweise und Warnungen an die Anwender gilt das zur Produkthaftung Ausgeführte.

Die Produktbeobachtungspflicht des Herstellers intelligenter Medizinprodukte wird konkretisiert durch die MPSV $^{63}$ und die Art. $83 \mathrm{ff}$. MDR/Art. $78 \mathrm{ff}$. IVDR. Aus dem Wissen des Herstellers um die objektive Unvermeidbarkeit von Programmierungsfehlern und das selbstständige Lernen KI-gestützter Software erwächst eine Pflicht zur besonders sorgfältigen Überwachung ihres Einsatzes. Das Ausmaß der Pflichten im Einzelfall richtet sich nach dem konkreten Gefährdungspotential des Produkts und seinem Einsatzgebiet ${ }^{64}$. Kann ein Fehler des intelligenten Medizinprodukts nicht vorhergesehen werden, trifft den Hersteller indes kein Verschulden; die Autonomie der KI kann zudem eine Unterbrechung des Kausalzusammenhangs bewirken $^{65}$. In beiden Fällen entfiele eine Haftung nach \823 Abs. 1 BGB. Dieser Problematik könnte nur mit einer Haftungserweiterung entsprechend der zum ProdHaftG diskutierten Anpassungen begegnet werden. Die Bundes-

44) Vgl. Taeger, NJW 2016, 3764, 3765 m.w. N.; Seehafer/Kohler, EuZW 2020, 213, 215; Oechsler, in: Staudinger, 2018, \$3 ProdHaftG, Rdnr. 85a.

45) Vgl. Droste, MPR 2018, 109, 113. Dazu auch Reusch, in: Kaulartz/ Braegelmann (Hrsg.), Rechtshandbuch Artificial Intelligence und Machine Learning, 2020, S. $124 \mathrm{ff}$.

46) Weißbuch, S. 18 f. Die Kommission erwägt, Anforderungen nur für KI-Systeme mit „hohem Risiko“ vorzusehen. S. auch BReg.SN Weißbuch, S. $9 \mathrm{f}$.

47) Vgl. Oechsler, in: Staudinger, 2018, $\$ 1$ ProdHaftG, Rdnr. 161

48) S. Kommissionsbericht, S. 14.

49) Vgl. Wagner, AcP 2017, 707, 750; Oechsler, in: Staudinger, 2018, \$2 ProdHaftG, Rdnr. 128.

50) So HEG-KI, Bericht zur Haftung, S. 6, Rdnr. 14.

51) So Teubner, AcP 2018, 155, 190.

52) Kommissionsbericht, S. 19; BReg.-SN Weißbuch, S. 25; HEGKI, Bericht zur Haftung, S. 6, Rdnr. 14.

53) So Kommissionsbericht, S. $19 \mathrm{f}$. Dazu auch Spindler, DB 2018, 41 , 49 f.; Teubner, AcP 2018, 155, 191 ff.; Katzenmeier, MedR 2019, 259, 270, Nachw. in Fn. 182; Wöbbeking, in: Kaulartz/Braegelmann (Hrsg.), Rechtshandbuch Artificial Intelligence und Machine Learning, 2020, S. $158 \mathrm{ff}$.

54) BReg.-SN Weißbuch, S. $26 \mathrm{f}$.

55) Teubner, AcP 2018, 155, $192 \mathrm{f}$.

56) Kommissionsbericht, S. 19f.; HEG-KI, Bericht zur Haftung, S. 9, Rdnr. 33; Teubner, AcP 2018, 155, 195.

57) Kommissionsbericht, S. 17 f. und 20; HEG-KI, Bericht zur Haftung, S. 6, Rdnr. 15

58) HEG-KI, Bericht zur Haftung, S. 7, Rdnrn. $20 \mathrm{ff}$.

59) BReg.-SN Weißbuch, S. 25f., 27.

60) BReg.-SN Weißbuch, S. 25.

61) Zu den Pflichtenkreisen im Bereich KI Reusch, in: Kaulartz/ Braegelmann (Hrsg.), Rechtshandbuch Artificial Intelligence und Machine Learning, 2020, S. $82 \mathrm{ff}$.

62) S. Oechsler, in: Staudinger, 2018, $\$ 3$ ProdHaftG, Rdnrn. $12 \mathrm{f}$; Wagner, in: MüKo/BGB, 7. Aufl. 2017, \$823, Rdnrn. 806, 818 ff.

63) So OLG Düsseldorf, PharmR 2012, 354, 363.

64) S. Spindler, DB 2018, 41, 48f.

65) Vgl. Denga, CR 2018, 69, 71; Feilemann, NZS 2018, 56. 
regierung hält ein unionsrechtliches Eingreifen in das deutsche Haftungsrecht indes auch dann nicht für erforderlich, wenn der schadensursächliche Gegenstand digital betrieben wird. Das deutsche Haftungsrecht gewähre auch beim Einsatz neuer Technologien umfassenden Schutz ${ }^{66}$.

Eine Haftung des Herstellers nach $\$ 823$ Abs. 2 BGB kann auf der Verletzung von Schutzgesetzen wie Art. 5 MDR/IVDR beruhen.

\section{b) Haftung des Anwenders bzw. behandelnden Arztes}

Verwendet ein Arzt ein KI-basiertes Medizinprodukt zur Diagnose oder Therapie und erleidet der Patient dadurch einen Schaden, kann der Behandelnde verschuldensabhängig zum Schadensersatz nach $\$ 280$ Abs. 1 i. V. mit $\$ 630$ a ff. BGB oder Deliktsrecht verpflichtet sein. Die Arzthaftung fußt auf der Nichtbeachtung medizinischer Sorgfaltspflichten, insbesondere des Gebots, die Behandlung dem aktuellen Erkenntnisstand von medizinischer Wissenschaft und Praxis anzupassen (\$630a Abs. 2 BGB).

Entspricht die Verwendung eines intelligenten Medizinprodukts nicht dem medizinischen Standard, kann das im Schadensfall haftungsbegründend wirken. Zum Standard wird eine Behandlungsmethode, wenn sie an einer ausreichend großen Patientenzahl erprobt, in der medizinischen Wissenschaft im Wesentlichen außer Streit steht und risikoärmer oder weniger belastend ist oder bessere Heilungschancen bietet ${ }^{67}$. KI hat durch ihre Fähigkeit zu lernen und sich stetig zu verbessern das Potenzial, präzisere Diagnosen und Therapieempfehlungen zu liefern als dies mit dem menschlichen Verstand möglich ist und wird deshalb voraussichtlich rasch zum medizinischen Standard avancieren $^{68}$. Bis dahin würde die Verwendung intelligenter Medizinprodukte nicht zwangsläufig einen groben Behandlungsfehler bedeuten, sofern der Arzt dem strengen Sorgfaltsmaßstab für neue Methoden genügt $t^{69}$.

Haftungsbegründende Fehler können in der Diagnosestellung, Therapieauswahl oder -durchführung, der Überwachung oder Nachsorge liegen, sofern der Behandler hierbei objektiv Falsches getan oder Richtiges unterlassen hat $^{70}$. Im Rahmen seiner Sorgfaltspflicht hat der Arzt dafür Sorge zu tragen, dass die von einer KI gelieferten und seiner Diagnose oder Therapie zugrunde gelegten Informationen richtig sind. Liegen Indizien für eine Fehlerhaftigkeit von Informationen vor, darf der Arzt sie nicht verwenden. Je gefährlicher eine fehlerhafte Information für den Patienten wäre, desto kritischer muss die ärztliche Bewertung $\operatorname{sein}^{71}$. Die regulatorischen Anforderungen an intelligente Medizinprodukte konkretisieren die ärztliche Sorgfaltspflicht: Der Einsatz eines nichtzertifizierten oder mangelhaften Produkts (\$11 MPDG) kann haftungsbegründend sein. Gleiches gilt im Falle des begründeten Verdachts der unmittelbaren oder mittelbaren Gefährdung der Sicherheit und Gesundheit der Patienten bei Verwendung des Produkts ( $\$ 12$ Nr. 1 MPDG). Aufgrund der technischen Komplexität der KI dürfte ein Arzt einen derart begründeten Verdacht vor einem schädigenden Ereignis allerdings nur selten haben ${ }^{72}$. Zum Teil wird vertreten, dass aufgrund der autonomen Entscheidungen eines intelligenten Medizinprodukts nur die Inbetriebnahme der Software als vorwerfbares Verhalten des Arztes dienen $\mathrm{kann}^{73}$. Um eine innovationsfeindliche „Veranlasserhaftung“ zu vermeiden, wird die analoge Anwendung des $\$ 278$ BGB auf das Verhalten der KI (Erfüllungsgehilfe) angeregt. Eine ärztliche Haftung würde selbst dann aber nicht bei jeder Diagnose entstehen, die sich im Nachhinein als falsch herausstellt, sondern nur bei einer schadensverursachenden Fehlentscheidung der KI, mag sie auch weder prognostizierbar noch erklärbar sein ${ }^{74}$.

Die Frage der ärztlichen Haftung hängt eng mit der Beweislast zusammen. Nach $\$ 630$ h Abs. 1 BGB wird ein Fehler des Behandelnden vermutet, wenn sich ein allgemeines Behandlungsrisiko verwirklicht hat, das für den Behandeln- den voll beherrschbar war und zur Verletzung des Patienten geführt hat. Der Patient hat den Nachweis zu erbringen, dass das voll beherrschbare Risiko und der erlittene Schaden kausal miteinander verknüpft sind, sofern nicht ein grober Behandlungsfehler gegeben ist und die Vermutung des $\$ 630 \mathrm{~h}$ Abs. 5 BGB eingreift ${ }^{75}$. Der Kausalitätsnachweis dürfte Patienten aufgrund der technischen Komplexität und der möglichen Vielzahl von Akteuren beim Einsatz intelligenter Medizinprodukte häufig nur schwer gelingen. Ist der Patient in die Bedienung des intelligenten Medizinprodukts eingebunden, kann auch ein Fehler auf seiner Seite ursächlich für den Schaden sein. Eine mögliche Abhilfe könnte die Protokollierung von Systemvorgängen schaffen, insbesondere der Vorgänge, die eine menschliche Interaktion umfassen ${ }^{76}$.

Eine Haftung des ärztlichen Verwenders intelligenter Medizinprodukte für autonome Entscheidungen der Software nach $\$ 831$ BGB unter dem Gesichtspunkt der KI als „,digitaler Verrichtungsgehilfe“ würde jedenfalls am Entlastungsbeweis nach $\int 831 \mathrm{~S}$. 2 BGB scheitern, wenn der Verwender seine Sorgfaltspflichten erfüllt hat. Im Übrigen scheint bereits fraglich, ob angesichts der fehlenden Rechtsfähigkeit und Weisungsgebundenheit autonom handelnder Software überhaupt eine Verrichtungsgehilfeneigenschaft in Betracht kommt ${ }^{77}$.

\section{c) Verordnungsvorschlag: Haftung des Betreibers} von KI-Systemen

In seinem Berichtsentwurf aus April 2020 schlägt das Europäische Parlament die Einführung einer Haftung des Betreibers von KI-Systemen vor. Die neuen Regelungen sollen die Form einer Verordnung annehmen und neben die verschuldensunabhängige Haftung des Herstellers mangelhafter KI-Systeme auf Grundlage des Produkthaftungsrechts (,zwei Säulen eines gemeinsamen Haftungsrahmens“) und das verschuldensabhängige Deliktsrecht der Mitgliedstaaten treten.

Betreiber soll die Person sein, die über die Verwendung des KI-Systems entscheidet, die Kontrolle über das Risiko ausübt und für die sein Betrieb einen Nutzen darstellt. Ein in das Schadensereignis involvierter Nutzer des KI-Systems bzw. Anwender i.S.d. MDR/IVDR würde haften, wenn er auch als Betreiber gilt. Im Fall intelligenter Medizinprodukte könnte der verantwortliche Arzt unter die Betreiberhaftung fallen. KI-Systeme mit hohem Risiko, d. h., solche, deren autonomer Betrieb ein signifikantes und nicht unwahrscheinliches Potenzial birgt, einen zufälligen, nicht vorhersehbaren schweren Schaden zu verursachen, sollen im Anhang zu der Verordnung aufgeführt werden und eine der Höhe nach begrenzte verschuldensunabhängige Haftung bewirken ${ }^{78}$. Betreiber solcher Hochrisikoanwendungen würden ferner einer

66) BReg.-SN Weißbuch, S. 28

67) Vgl. Kern/Rehborn, in: Laufs/dies., Handbuch des Arztrechts, 5. Aufl. 2019, \$97, Rdnr. 50; Dierks, in: Jähn/Nagel (Hrsg.), eHealth, 2004, 274, 276.

68) Vgl. Katzenmeier, MedR 2019, 259, 268

69) BGHZ 168, 103, 105 f.; Katzenmeier, NJW 2006, 2738, 2740.

70) Vgl. Kern/Rehborn, in: Laufs/dies., Handbuch des Arztrechts, 5. Aufl. 2019, \$97, Rdnrn. $17 \mathrm{ff}$.

71) S. Droste, MPR 2018, 109, 112; Bördner, GuP 2019, 131, 133 f.

72) So Droste, MPR 2018, 109, 112; Bördner, GuP 2019, 131, 134.

73) S. Teubner, AcP 2018, 155, $185 \mathrm{f}$.

74) Lindenberg, ZMGR 2020, 12, 16; Teubner, AcP 2018, 155, $185 \mathrm{ff}$.

75) Die gesetzliche Vermutung hat nach $\$ 292$ S. 1 ZPO die Wirkung einer Beweislastumkehr. Dazu Katzenmeier, NJW 2019, 1769, 1773.

76) Vgl. Droste, MPR 2018, 109, 113f.

77) S. Teubner, AcP 2018, 155, 190; Reusch, in: Kaulartz/Braegelmann (Hrsg.), Rechtshandbuch Artificial Intelligence und Machine Learning, 2020, S. 141. Die Verrichtungsgehilfeneigenschaft bejaht etwa Denga, CR 2018, 69, 76

78) Ebenso HEG-KI, Bericht zur Haftung, S. 6, Rdnrn. 9, 10. Hierfür plädiert auch Spindler, DB 2018, 41, 50. 
gesetzlichen Versicherungspflicht unterliegen. Für Betreiber anderer KI-Systeme soll dagegen eine verschuldensabhängige Haftung gelten. Allerdings würde das Verschulden vermutet und dem Betreiber stünde nur ein auf bestimmte Gründe gestützter Entlastungsbeweis zu.

Allein die Zahl der Änderungsanträge zum Entwurf des Rechtsausschusses $(430)^{79}$ zeigt, dass die Diskussion über die neuen Regelungen für die Betreiberhaftung erst am Anfang steht ${ }^{80}$.

\section{Datenschutz}

Der Einsatz Künstlicher Intelligenz - von der Konfiguration über das Training von KI-Systemen bis hin zu einem erfolgreichen Betrieb - ist abhängig von der Verwendung großer Datenmengen (Big Data). Hiermit sind zwangsläufig datenschutzrechtliche Fragen verbunden. Der Beitrag zeigt die besonders relevanten Punkte im Anwendungsbereich der DSGVO ${ }^{81}$ im Überblick auf:

- Eine Datenverarbeitung mit Hilfe von Algorithmen unterfällt nur dann dem Anwendungsbereich der DSGVO, wenn sie personenbezogene Daten i.S.d. Art. 4 Abs. 1 Nr. 1 als Ein- oder Ausgabedaten verwendet (Art. 2 Abs. 1); nicht reguliert ist dagegen die Verwendung anonymisierter Daten (Erwg. 26). Von großer praktischer Relevanz ist daher im Ausgangspunkt, ob die für den KI-Einsatz erforderlichen Daten durch die irreversible Entfernung des Personenbezugs anonymisiert werden können ${ }^{82}$. Alternativ empfiehlt es sich, die Möglichkeit der Pseudonymisierung der Daten zu prüfen, um zumindest gewisse Erleichterungen $\mathrm{zu}$ genießen ${ }^{83}$. Adressat der datenschutzrechtlichen Regelungen ist der Verantwortliche i.S.d. Art. 4 Nr. 7, d.h., derjenige, der über Zwecke und Mittel der Verarbeitung entscheidet. Das können Hersteller wie auch Anwender intelligenter Medizinprodukte $\operatorname{sein}^{84}$.

- Die zentralen datenschutzrechtlichen Grundsätze gem. Art. 5 Abs. 1 wie Einwilligung, Transparenz, Zweckbindung, Datenminimierung und Datenrichtigkeit können bei der Verwendung großer Datenmengen zum Einsatz von KI an ihre Grenzen stoßen, zumal wenn es um die Verarbeitung besonders geschützter Gesundheitsdaten geht (vgl. Art. 4 Nr. 15, 9 Abs. 2 Buchst. a) ${ }^{85}$.

- Schon im Stadium der KI-Generierung für das intelligente Medizinprodukt hat eine Datenschutzfolgenabschätzung nach Art. 35 zu erfolgen ${ }^{86}$. In der Phase des Einsatzes von KI werden die Informationspflichten nach Art. 13, 14 und das Auskunftsrecht des Betroffenen nach Art. 15 relevant $^{87}$, es sei denn, es liegen nur pseudonymisierte Daten vor.

- Der Hersteller intelligenter Medizinprodukte ist an die datenschutzrechtlichen Vorgaben im technischen Design der Verarbeitung (privacy by design) und in den zugehörigen Unternehmensprozessen (privacy by default) gebunden (Art. 25).

- Ob die Datenverarbeitung intelligenter Medizinprodukte den Anforderungen des Art. 22 an (ausschließlich) automatisierte Entscheidungen unterliegt, hängt von den Umständen des Einzelfalls ab. Die Regelung gilt von vornherein nicht für lediglich durch Algorithmen unterstützte menschliche Entscheidungen, wenn also ein Mensch die Befugnis hat und tatsächlich in der Lage ist, die ,,künstliche“"Entscheidung zu überprüfen oder aus bloßen Vorschlägen auszuwählen ${ }^{88}$.

- Die DSGVO determiniert nicht, ob und unter welchen Voraussetzungen benannte Stellen Zugriffsrechte auf personenbezogene Daten haben, was im Rahmen der Konformitätsbewertungsverfahren von Relevanz sein wird ${ }^{89}$.

Bei der Konzeption der DSGVO standen weder Big Data noch KI im Fokus. Ob die Verordnung den Einsatz neuartiger Analyseverfahren im Sinne eines innovationsfreundlichen Regimes regeln kann, wird von ihrer Auslegung durch Aufsichtsbehörden und Gerichte abhängen. Die Datenschutzaufsichtsbehörden des Bundes und der Länder (DSK) haben sich bereits in Form von Entschließungen zur KI positioniert ${ }^{90}$. Zuversichtlich stimmt die Äußerung der DSK $^{91}$, ,dass der Einsatz von KI-Systemen und der Datenschutz keine zwingenden Gegensätze sind. Die Chancen und neuen Möglichkeiten des Einsatzes von KI-Systemen werden durch einen modernen Datenschutz nicht verhindert." Sollten Datenschutzvorgaben, die nicht unbedingt notwendig für den Schutz der Privatsphäre sind, die Entwicklung von KI-Anwendungen (doch) einschränken oder gar behindern, ist der europäische Gesetzgeber zu Nachbesserungen an der DSGVO oder der Schaffung spezialgesetzlicher Regelungen aufgerufen ${ }^{92}$.

\section{Ausblick}

Die Diskussion zu der digitalen Zukunft und dem Rechtsrahmen für den Einsatz intelligenter Medizinprodukte ist angestoßen. Mit ihrem Weißbuch und dem begleitenden Bericht präsentierte die Kommission wichtige Impulse für den künftigen Umgang mit KI und stellte politische $\mathrm{Op}$ tionen mit dem Ziel vor, die Entwicklung und Nutzung der KI in einem europäischen Regulierungsrahmen zu fördern. Auf Grundlage des Konsultationsverfahrens und

79) Abrufbar unter https://www.europarl.europa.eu/committees/ de/juri/documents/latest-documents, Zugriff am 23.8.2020.

80) Eher kritisch zu einer Betreiberhaftung Wöbbeking, in: Kaulartz/ Braegelmann (Hrsg.), Rechtshandbuch Artificial Intelligence und Machine Learning, 2020, S. $159 \mathrm{ff}$.

81) Verordnung (EU) 2016/679 zum Schutz natürlicher Personen bei der Verarbeitung personenbezogener Daten, zum freien Datenverkehr und zur Aufhebung der Richtlinie 95/46/EG.

82) Zur Anonymisierung bei Big Data Valkanova, in: Kaulartz/Braegelmann (Hrsg.), Rechtshandbuch Artificial Intelligence und Machine Learning, 2020, S. $340 \mathrm{ff}$. und Paal, ebd., S. $437 \mathrm{ff}$.

83) Dazu Arning/Rothkegel, in: Taeger/Gabel, DSGVO, 3. Aufl. 2019, Art. 4, Rdnrn. $119 \mathrm{fff}$.

84) Dazu Skistims, in: Kaulartz/Braegelmann (Hrsg.), Rechtshandbuch Artificial Intelligence und Machine Learning, 2020, S. 352 f. und Paal, ebd., S. 440 und Brink/Bäßler/Groß-Karreis, ebd., S. $492 \mathrm{f}$

85) Dazu Katzenmeier, MedR 2019, 259, 263 m.w. N.; Dettling, PharmR 2019, 633, 641; Gausling, PinG 2019, 61, 65 ff.; von Walter, K\&R 2019, 21, 23 ff.; Paal, in: Kaulartz/Braegelmann (Hrsg.), Rechtshandbuch Artificial Intelligence und Machine Learning, 2020, S. $428 \mathrm{ff}$.

86) Mit Privilegierung pseudonymisierter Daten. S. Gausling, PinG 2019, 61, 67f.; Frost, MPR 2019, 117, $123 \mathrm{f}$.

87) Näher dazu Gausling, in: Kaulartz/Braegelmann (Hrsg.), Rechtshandbuch Artificial Intelligence und Machine Learning, 2020, S. $379 \mathrm{fff}$.

88) S. Spindler, DB 2018, 41, 46. Zu Art. 22 ausführlich von Walter, in: Kaulartz/Braegelmann (Hrsg.), Rechtshandbuch Artificial Intelligence und Machine Learning, 2020, S. $391 \mathrm{ff}$.

89) Art. 58 Abs. 1 regelt allein aufsichtsbehördliche Zugriffsrechte. Dazu näher Hacker, NJW 2020, 2142, 2144.

90) Hambacher Erklärung zur Künstlichen Intelligenz v. 3. 4.2019, https://www.datenschutzkonferenz-online.de/media/ en/20190405_hambacher_erklaerung.pdf; Empfehlungen für eine datenschutzkonforme Gestaltung von KI-Systemen, https:// www.datenschutzkonferenz-online.de/media/en/20191106_ entschlie\%C3\%9Fung_KI_DSK.pdf, einschließlich Positionspapier der DSK zu empfohlenen technischen und organisatorischen Maßnahmen bei der Entwicklung und dem Betrieb von KI-Systemen v. 6.11.2019, https://www.datenschutzkonferenzonline.de/media/en/20191106_positionspapier_kuenstliche_intelligenz.pdf; Zugriff am 23.8.2020.

91) DSK Empfehlungen für eine datenschutzkonforme Gestaltung von KI-Systemen v. 6.11.2019.

92) Vgl. Bitkom, Künstliche Intelligenz, 2017, S. 133, 139, abrufbar unter https://www.bitkom.org/sites/default/files/file/import/171012KI-Gipfelpapier-online.pdf, Zugriff am 23. 8.2020. 
eines „Inception Impact Assessment“ ist im ersten Quartal 2021 ein Vorschlag für einen künftigen KI-Rechtsrahmen $\mathrm{zu}$ erwarten, der nach heutigem Stand u.a. eine Revision der Produkthaftungsrichtlinie sowie Anforderungen an die Entwicklung und den Einsatz rechtmäßiger und vertrauenswürdiger KI umfassen wird ${ }^{93}$. Es bleibt zu hoffen, dass der europäische Gesetzgeber den Spagat zwischen Innovationsförderung und Ordnungspolitik für KI i.S. der Innovation nutzt und Anpassungen des geltenden Rechtsrahmens an die Erfordernisse des Einsatzes von KI in der Medizin auf die absolut notwendigen Eingriffe beschränkt, ohne in einer innovationshemmenden Überregulierung zu enden. Die Hoffnung wird u. a. dadurch gestärkt, dass die Kommission jüngst ankündigte, den Einsatz einer KIbasierten Software in zehn Krankenhäusern in Europa zu fördern, die in weniger als einer Minute CT Scans auf eine COVID-19 Erkrankung analysiert ${ }^{94}$.
Mit dem deutschen Ratsvorsitz im zweiten Halbjahr 2020 wird auch die Bundesregierung bei der Förderung von KI eine wichtige Rolle spielen. So steht u. a. die Entwicklung eines digitalen Frühwarnsystems auf dem Programm, um noch besser auf künftige der COVID-19 Pandemie vergleichbare Herausforderungen vorbereitet und handlungsfähig zu sein ${ }^{95}$.

93) S. https://ec.europa.eu/info/law/better-regulation/have-your-say/ initiatives/12527-Requirements-for-Artificial-Intelligence, Zugriff am 23.8.2020.

94) Vgl. Kommission, Pressebericht v. 19.5.2020, abrufbar unter https:// ec.europa.eu/digital-single-market/en/news/using-ai-fast-and-effectively-diagnose-covid-19-hospitals, Zugriff am 23.8.2020.

95) Programm des deutschen Vorsitzes im Rat der EU, S. 5, abrufbar unter https://www.eu2020.de/blob/2360248/e0312c50f91 0931819ab67f630d15b2f/pdf-programm-en-data.pdf, Zugriff am 23. 8.2020 .

\section{Masernimpfpflicht, Maskenpflicht und unrichtige Gesundheitszeugnisse}

\section{Sven Henseler}

\begin{abstract}
Masernimpfpflicht und das Tragen einer Mund-Nasen-Bedeckung können aus medizinischen Gründen suspendiert werden. Stellen $\ddot{A r z t} t^{\star}$ innen diesbezüglich ein unrichtiges Gesundheitszengnis aus, machen sie sich nach $\$ 278$ StGB strafbar. Sie handeln sorgfaltsund standeswidrig, daher kann das Verhalten auch Anknüpfungspunkt für eine fahrlässige Körperverletzung (bzw. Tötung) sein und zum Widerruf der Approbation führen.
\end{abstract}

\section{Einleitung}

Mit dem Gesetz für den Schutz vor Masern und zur Stärkung der Impfprävention (Masernschutzgesetz) wurden mit Wirkung zum 1.3.2020 ${ }^{1}$ Personen verpflichtet, die nach dem 31.12.1970 geboren sind und in einer Gemeinschaftseinrichtung $^{2}$ betreut ${ }^{3}$ werden oder tätig sind ${ }^{4}$, einen ausreichenden Impfschutz gegen Masern oder $\mathrm{ab}$ der Vollendung des ersten Lebensjahres eine Immunität gegen Masern aufzuweisen $(\$ 20$ Abs. 8 S. 1 IfSG $)^{5}$. Eine Ausnahme gilt für Personen, die aufgrund einer medizinischen Kontraindikation nicht geimpft werden können (\$20 Abs. 8 S. 4 IfSG).

Die Reaktionen im Internet belegen, dass das Gesetz Wasser auf die Mühlen von Impfgegnern, Impfkritikern, Verteidigern der Menschenwürde und selbsternannten Medizinjournalisten war $^{6}$. In Foren wird sich über Umgehungsstrategien ausgetauscht. In einem Eilverfahren hat das $\mathrm{BVerfG}^{7}$ einen Antrag auf Erlass einer einstweiligen Anordnung ${ }^{8}$ abgelehnt. Dies sagt noch nichts über die Vereinbarkeit mit dem Verfassungsrecht aus ${ }^{9}$, sondern stellt lediglich fest, dass das Interesse an einer vorläufigen Aussetzung des Gesetzes hinter den Interessen an einer vorläufigen Weitergeltung zurücktritt ${ }^{10}$.

Rechtsanwalt Prof. Dr. iur. Sven Henseler,

Diplom-Finanzwirt (FH),

Inhaber einer Qualifikationsprofessur für Strafrecht,

Strafprozessrecht, Wirtschaftsstrafrecht und Steuerstrafrecht,

EBS Universität für Wirtschaft und Recht,

Gustav-Stresemann-Ring 3, 65189 Wiesbaden, Deutschland
Die Corona-Pandemie hat auch zu Vorschriften zum Schutz von anderen geführt. In den Landesverordnungen finden sich Regelungen über das verpflichtende Tragen von Mund-Nasen-Bedeckungen in bestimmten Situationen (Fahrzeuge des öffentlichen Personennah- und -fernverkehrs, Verkaufsstätten und ähnliche Einrichtungen, etc. $)^{11}$. Ausgenommen sind Personen, die aufgrund einer gesundheitlichen Beeinträchtigung oder Behinderung kei-

1) BGBl. I S. 148 .

2) $\$ 33$ Nr. 1 bis 3 IfSG: Kindertageseinrichtungen und Kinderhorte, nach $₫ 43$ Abs. 1 SGB VIII erlaubnispflichtige Kindertagespflege, Schulen und sonstige Ausbildungseinrichtungen.

3) Die Verpflichtung gilt auch für Personen, die bereits vier Wochen in einem Heim ( $\$ 33 \mathrm{Nr} .4$ IfSG) betreut werden oder in einer Einrichtungen zur gemeinschaftlichen Unterbringung von Asylbewerbern, vollziehbar Ausreisepflichtigen, Flüchtlingen und Spätaussiedlern (\$36 Abs. $1 \mathrm{Nr}$. 4 IfSG) untergebracht sind.

4) Auch Tätige in weiteren Einrichtungen nach den $\$ 23$ Abs. 1 und $\$ 36$ Abs. 1 IfSG.

5) Ausführlich zu den Einrichtungen und Pflichten: Rixen, NJW 2020, 647, 647f.

6) Quelle: Internet oder Youtube, um die Zitierung der selbsternannten „Experten“ aufzugreifen.

7) BVerfG, NJW 2020, $1946 \mathrm{ff}$.

8) Antrag und Verfassungsbeschwerde betreffen ein Elternpaar und ihre beiden ungeimpften Kinder, bei denen weder eine Kontraindikation noch eine Immunität vorliegen.

9) BVerfG, NJW 2020, 1946, 1947: „Die Verfassungsbeschwerde ist zumindest nicht von vornherein unzulässig oder offensichtlich unbegründet".

10) Zur Frage der Verfassungsmäßigkeit des $\$ 20$ Abs. 8 S. 3 IfSG: Schaks, MedR 2020, $201 \mathrm{ff}$; vgl. zur Verfassungsmäßigkeit insgesamt die Verweise in Fn. 53 bei Rixen, NJW 2020, 647, 651.

11) Beispielsweise: $\$ 1$ Abs. 6 S. 1 und $\$ 3$ Abs. 1 S. 1 Verordnung zur Beschränkung von sozialen Kontakten und des Betriebes von Einrichtungen und von Angeboten aufgrund der Corona-Pandemie (Corona-Kontakt-und Betriebsbeschränkungsverordnung) i.d.F. der am 6.7.2020 in Kraft tretenden Änderungen durch Art. 3 der Fünfzehnten Verordnung zur Anpassung der Verordnungen zur Bekämpfung des Corona-Virus v. 1.7.2020 (GVB1. S. 473). 